http://jmscr.igmpublication.org/home/ ISSN (e)-2347-176x ISSN (p) 2455-0450 crossref DOI: https://dx.doi.org/10.18535/jmscr/v8i2.03

\title{
Rare Case of Polycythemia Vera Presenting As Portal Vein and Splenic Vein Thrombosis - A Case Report
}

\author{
Authors \\ Revathi Potnuru ${ }^{1 *}$, Ramana Murthy S.V ${ }^{2}$, Padma Guduri ${ }^{3}$ \\ GSL medical college, Rajanagaram, Rajahmundry 533296 Andhra Pradesh, India \\ *Corresponding Author \\ Revathi Potnuru
}

\begin{abstract}
Polycythemia vera is one of the chronic myeloproliferative neoplasms. It is a clonal hematopoietic stem cell disorder in which phenotypically normal red cells, granulocytes, and platelets accumulate in the absence of a recognizable physiologic stimulus. It occurs in 2.5 per 100,000 persons, sparing no adult age group. Approximately one tenth cases present before age 40 years. Mutation in the JAK2V617F tyrosine kinase appears to play a central role in the pathogenesis of PV. It can cause venous or arterial thrombosis of any vessel, but cerebral, cardiac, and mesenteric vessels are most commonly involved. Here we report a case of 29 year old male patient, who was admitted with abdominal distension, icterus, pedal edema and was found to have portal vein and splenic vein thrombosis due to polycythemia vera on evaluation. It is important to identify such cases because it may cause death due to thrombosis or it may transform into AML.
\end{abstract}

\section{Introduction}

Polycythemia vera (PV) is the most common chronic myeloproliferative disorder with an incidence of 2.5 case per one lakh population. It is a clonal disorder involving multipotent hematopoietic progenitor cell in which phenotypically normal red cells, granulocytes and platelets accumulate in the bone marrow in the absence of a physiologic stimulus. PV is characterized by the expression of a JAK2 mutation, V617F that causes constitutive activation of tyrosine kinase that is essential for the function of the erythropoietin and thrombopoietin. PV should be suspected in any patient with increased red blood cell mass or increased haemoglobin/hematocrit. It should also be suspected in patients with portal, splenic, or mesenteric vein thrombosis. WHO criteria for the diagnosis of PV includes increased haemoglobin $(>16.5 \mathrm{~g} / \mathrm{dl}$ in men or $>16 \mathrm{~g} / \mathrm{dl}$ in women), hematocrit ( $>49 \%$ in men or $>48 \%$ in women), or other evidence of increased red cell volume, bone marrow biopsy showing trilineage hypercellularity, JAK2 V617F mutation and serum erythropoietin level below the reference range for normal.

\section{Case Report}

A 29 year old male patient who is a non-alcoholic was admitted with complaints of pain, distension and tightness of the abdomen of 2 days duration. The pain was diffuse and was associated with generalized body pains, swelling of both lower limbs and decreased urine output. There was yellowish discolouration of eyes with altered sleep pattern and loss of appetite. On clinical 
examination- patient had icterus and bilateral pitting pedal edema upto knees. Abdomen was soft, distended with massive ascites, liver and spleen could not be palpated. Patient was drowsy but coherent and oriented and responding to verbal stimuli, Asterixis was present and bilateral plantars were flexors. His blood investigations revealed $\mathrm{Hb}-$ 20 gm/dl, PCV-64\%, RBC-8.8 million/cumm, TLC - 32,100 cells/cumm, Platelets-9.3lakh/cumm, LFTTotal bilirubin-6mg/dl, direct bilirubin- $1 \mathrm{mg} / \mathrm{dl}$, with elevated liver enzymes, serum total proteins $-4.9 \mathrm{~g} / \mathrm{dl}$ and albumin-2.8g/dl, PT INR-7.0, Blood urea$110 \mathrm{mg} / \mathrm{gl}$, serum creatinine- $1.5 \mathrm{mg} / \mathrm{dl}$, viral markersnon-reactive, serum uric acid-10.3mg/dl. His USG and CECT abdomen revealed cirrhosis of liver with portal hypertension, portal vein and splenic vein thrombosis, splenomegaly with multiple splenic infarcts, massive ascites. Erythropoietin-3.9Miu/ml, JAK2 mutation-positive, BCR-ABL-negative and bone marrow aspiration showed hypercellular marrow with megaloblastic and erythroid hyperplasia and increased megakaryocytes-features suggestive of myeloproliferative disorder-possibly polycythemia vera. The initial diagnosis in this case was cirrhosis of liver with portal vein thrombosis and the cause of portal vein thrombosis was found to be polycythemia vera after thorough haematological investigations. Patient was started on chemotherapy with Hydroxyurea and Ruxolitinib and there was symptomatic improvement at the time of discharge and patient was advised regular follow up.

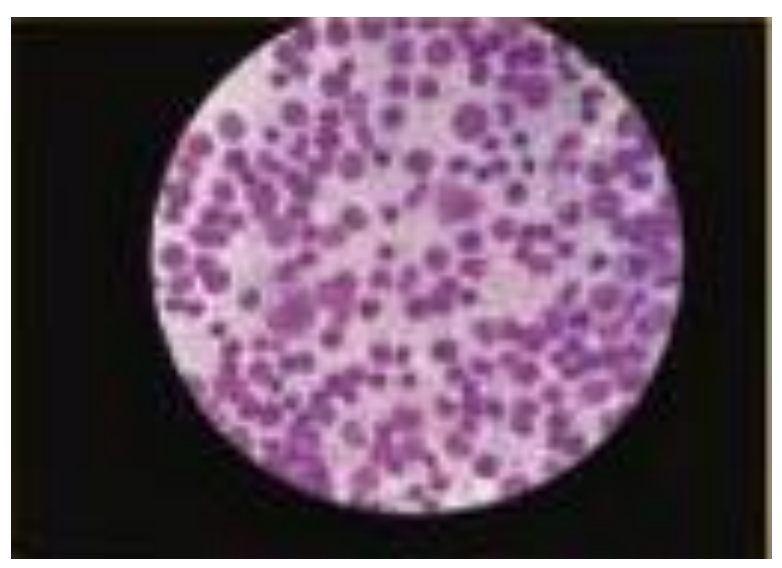

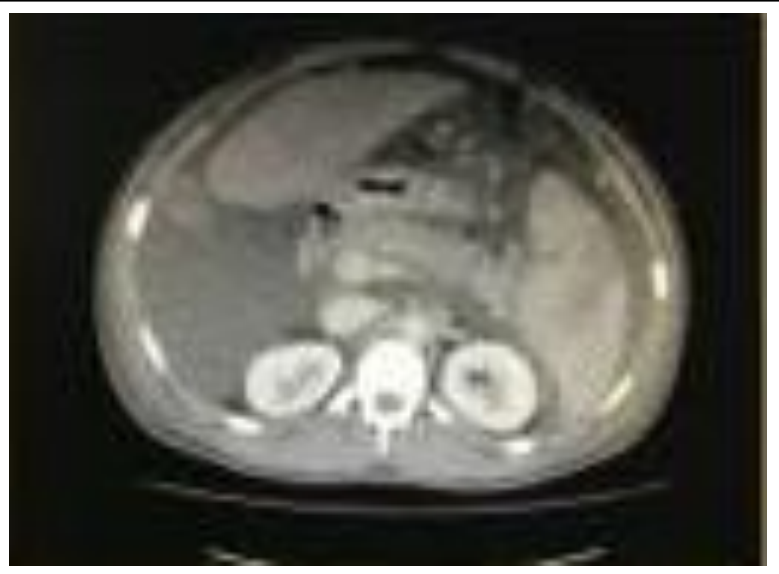

\section{Discussion}

Myeloproliferative disorders are one of the causes for portal vein thrombosis and they have emerged as a leading systemic cause of portal vein thrombosis during the last several years. Polycythemia vera is a clonal haemopoietic stem cell disorder involving hyperplasia of all three major cell lineages. The median age of onset is $55-60$ years $^{[1]}$ with cases under the age of 20 being very rare. PV has an incidence of 2.5 case per one lakh population. $\mathrm{PV}$ is characterized by the expression of a JAK2 mutation, $\mathrm{V} 617 \mathrm{~F}$ that causes constitutive activation of tyrosine kinase that is essential for the function of the erythropoietin and thrombopoietin. Uncontrolled erythrocytosis causes hyperviscosity, leading to neurologic symptoms like vertigo, tinnitus, headache, visual disturbances.In some patients, venous or arterial thrombosis may be the presenting manifestation of PV. Mesenteric, hepato-portal or splenic vein thrombosis should always raise suspicion of PV as a possible cause ${ }^{[2]}$. Digital ischemia, easy bruising, epistaxis, acid-peptic disease, or gastrointestinal hemorrhage may occur due to vascular stasis or thrombocytosis. Erythromelalgia causing erythema, burning, and pain in the extremities is another complication of thrombocytosis in PV due to increased platelet stickiness. Due to large turnover of hematopietic cells, hyperuricemia with secondary gout, uric acid stones, and symptoms due to hypermetabolism can also occur.

WHO criteria ${ }^{[3]}$ for the diagnosis of PV includes 3 major criteria-increased haemoglobin $(>16.5 \mathrm{~g} / \mathrm{dl}$ in men or $>16 \mathrm{~g} / \mathrm{dl}$ in women), hematocrit $(>49 \%$ in 
men or $>48 \%$ in women), or other evidence of increased red cell volume, bone marrow biopsy showing trilineage hypercellularity, JAK2 V617F mutation and one minor criterion-serum erythropoietin level below the reference range for normal. Diagnosis of PV requires either all 3 major criteria, or the first 2 major criteria and the minor criterion Treatment of PV includes Phlebotomy to maintain the hematocrit $<45 \%$, low dose aspirin 40 $100 \mathrm{mg}$ once or twice daily, unless there is a contraindication to its use, cytoreductive therapy with Hydroxy urea $15 \mathrm{mg} / \mathrm{kg} /$ day or Busulfan. For refractory case, Ruxolitinib, a janus kinase inhibitor upto $20 \mathrm{mg}$ twice daily.

The initial diagnosis in our case was cirrhosis of liver with portal vein thrombosis and the cause of portal vein thrombosis was found to be polycythemia vera after thorough haematological investigations. Patient was started on chemotherapy with Hydroxyurea and Ruxolitinib and there was symptomatic improvement at the time of discharge.

\section{Conclusion}

In conclusion, this is a rare case of polycythemia vera presented as cirrhosis with portal vein and splenic vein thrombosis. Diagnosis and treatment of underlying haematological condition is important in this case because transformation to AML or MDS is a major cause of death in PV. In a large international study, approximately 7 percent of patients with PV developed AML/MDS within 20 years $^{[4]}$.

\section{References}

1. Tefferi, A., JAK2 mutations in polycythemia vera-molecular mechanisms and clinical applications. N Engl J Med, 2007;356:444-5.

2. Pierre R, Vardiman JW, Imbert M, Brunning RD, Thiele J, Flandrin G. Polycythemia Vera. In: World Health Organization classification of tumors: pathology and genetics of tumors of haematopoitic and lymphoid tissues,2001;32-34.

3. Spivak JL, Silver RT. The revised World Health Organization diagnostic criteria for polycythemia vera, essential thrombocytosis, and primary myelofibrosis: an alternative proposal. Blood 2008;112:231.

4. Tefferi A, Guglielmelli P, Larson DR, et al Long-term survival and blast transformation in molecularly annotated essential thrombocythemia, polycythemia vera, and myelofibrosis. Blood 2014;124:2507. 